\title{
Experimental and Numerical Investigations of Flow Incidence Effects on Surface Pressure Distributions of Axial Compressor Blades
}

\author{
Reza Taghavi Zenouze, Farzin Ghanadi ${ }^{*}$ and Majed Etemadi \\ Iran University of Science and Technology, Tehran, Iran
}

\begin{abstract}
In the present research work, intensive investigations were performed on axial compressor blades from experimental and numerical point of views and results are confined to variations of surface pressure distributions versus flow incidence. Test model was a cascade consisting of three rotor blades of an axial compressor with profile geometry introduced as NGTE 10C4/30C50 section. Tests were carried out in an open circuit wind tunnel of blowing type. Tunnel walls were modified somehow to fit the proposed intentions. In this respect, a suitable test section together with a secondary nozzle were designed and manufactured. These were connected to the exit section of the original wind tunnel. The Reynolds number was changed from 250,000 to 410,000 based on the blade chord length. 30 pressure tappings were mounted evenly all around the surface of the middle profile both on the pressure and suction surfaces. A suitable data accusation system was used to log the surface static pressures under different conditions. In parallel to the wind tunnel tests flow characteristics were studied using computational fluid dynamics (CFD) technique. Reynolds averaged Navier-Stokes equations were solved using realizable K- $\varepsilon$ turbulence modeling. Finally, numerical results were compared to those obtained through the tests which showed close agreements and conclusion shows that the relationship between incidence variations and pressure coefficient changes.
\end{abstract}

Key words: Compressor cascade flows, surface pressure distribution, incidence effects, turbulence modelling.

\section{Introduction}

Cascade data are essential in design process of any turbomachine blades. These data consist of variations of losses, pressure rise, surface pressure distributions and outlet flow angle in terms of Reynolds number, inlet Mach number and flow incidence [1, 2]. In this paper the performance of NGTE $1065 \mathrm{c} 40$ compressor blade sections in cascade has been investigated systematically in a low-speed cascade tunnel. In this paper the performance of NGTE $1065 \mathrm{c} 40$ compressor blade sections in cascade for various incidences has been investigated. Compressor designers use cascade data not only for selection of profile geometry to suit their predicted on design performance, but also to meet off design conditions. Blade incidence may change due to many factors. These factors mainly consist of

Corresponding author: Farzin Ghanadi, M.Sc., research field: aerodynamics of compressors. E-mail: farzinghanadi@mecheng.iust.ac.ir. rotational speed of the compressor axis and inlet flow conditions. Design point in majority of turbomachines does not necessarily correspond to zero incidence. As a result, cascade data at various incidences would be vital during design performance [3].

The design of an axial-flow compressor of high performance involves three-dimensional high-speed flow of compressible viscous gases through successive rows of closely spaced blades. No adequate theoretical solution for this complete problem has yet appeared nor considering the complexity of the problem does it seem likely that complete relationships will be established for some time. Various aspects of the problem have been treated theoretically, and the results of those studies are quite useful in design calculations. However, all such studies have been based on idealized flow, neglecting effects of one or more such physical realities as compressibility, finite blade spacing and viscosity. Consideration of viscosity effects has been particularly 
difficult. Therefore, it appears that in spite of advances in theoretical methods, theory must be supplemented by experimental data for some time to come [4]. Some of the required information can be obtained only by experiment in single-stage and multistage compressors. However, much of the information can be obtained more easily by isolating the effects of each parameter for detailed measurement. The effects of inlet angle, blade shape, angle of attack, solidity on the turning angle and drag produced can be studied by tests of compressor blades in two-dimensional cascade tunnels. Cascade tests can provide many basic data concerning the performance of compressors under widely varying conditions of operation with relative ease, rapidity and low cost. A number of successful high-speed axial-flow compressors have been designed using low-speed cascade data directly. However, a more refined procedure would use cascade data, not as the final answer, but as a broad base from which to work out the three-dimensional relations. The main function of a compressor cascade is to raise the static pressure of the fluid across it. This should be achieved with minimum stagnation pressure loss or drag. Thus the compressor cascade behaves like an adiabatic diffuser. The static pressure rise for isentropic flow is given by these Eqs. (1)-(3):

$$
\begin{gathered}
\Delta p_{s}=p_{2 s}-p_{1}=\frac{1}{2} \rho\left(c_{1}^{2}-c_{2 s}^{2}\right) \\
\Delta p_{s}=\frac{1}{2} \rho c_{1}^{2}\left(1-\frac{\cos ^{2} \alpha_{1}}{\cos ^{2} \alpha_{2}}\right) \\
\Delta p_{s}=\frac{1}{2} \rho c_{1}^{2}\left(1-\frac{c_{2 s}^{2}}{c_{1}^{2}}\right)
\end{gathered}
$$

where $\alpha_{1}$ and $\alpha_{2}$ are air angles in inlet and outlet respectively, $c$ is air velocity. The pressure rise through the cascade can be expressed as a dimension less quantity known as the pressure or pressure recovery coefficient [5]. Thus the ideal pressure recovery coefficient for the cascade is given by:

$$
C_{p s}=\frac{\Delta p_{s}}{\frac{1}{2} \rho c_{1}^{2}}=1-\frac{\cos ^{2} \alpha_{1}}{\cos ^{2} \alpha_{2}}
$$

For an actual flow the static pressure rise is given by Eqs. (5)-(7):

$$
\begin{gathered}
\Delta p_{a}=p_{2}-p_{1}=\Delta p_{s}-\Delta p_{0} \\
\Delta p_{\alpha}=\frac{1}{2} \rho c_{1}^{2}\left(1-\frac{\cos ^{2} \alpha_{1}}{\cos ^{2} \alpha_{2}}\right)-\Delta p_{0} \\
C_{p \alpha}=\frac{\Delta p_{a}}{\frac{1}{2} \rho c_{1}^{2}}=\left(1-\frac{\cos ^{2} \alpha_{1}}{\cos ^{2} \alpha_{2}}\right)-\frac{\Delta p_{0}}{\frac{1}{2} \rho c_{1}^{2}}
\end{gathered}
$$

\section{Test Facility and Instrumentation}

\subsection{The Wind Tunnel}

Almost all typical NGTE low-speed cascade tunnels discharge to the atmosphere and are powered by variable-speed centrifugal or axial blowers [6]. Honeycombs are used to remove any swirl from air and screens are installed in settling chamber to insure a uniform velocity distribution entering test section. In this research solid side wall open circuit blower tunnel, limit layer and turbulence TE44 of the Mechanical Engineering Department, Iran University of Science and Technology, was used for the present investigation.

The tunnel work room is rectangular parallelepiped with the next dimensions: $(\mathrm{L}=0.85 \mathrm{~m}, \mathrm{~B}=0.14 \mathrm{~m}$ and $\mathrm{H}=0.457 \mathrm{~m}$ ), and having the following characteristics: $\mathrm{P}=25 \mathrm{kw}$ and These can work singular or together, assuring, in the experimental work area, maximum speeds of approximate $52 \mathrm{~m} / \mathrm{s}$. Tunnel walls were modified somehow to fit the proposed intentions. A suitable test section together with a secondary nozzle were designed and manufactured. These were connected to the exit section of the original wind tunnel.

\subsection{Description of Airfoils}

The three tested blades in this research (NGTE10C4/30C50) have similar cascade geometry (chord, aspect ratio, solidity, stagger angle, and camber angle), the first two digits (10) denote the maximum thickness in percent chord; $\mathrm{C} 4$ indicates a thickness distribution; 30 indicates camber angle in degree; $\mathrm{C}$ refers to the type of mean line, in this case, circular arc; and 50 is the distance of the point of maximum camber from the leading edge in percent chord [7]. 
Table 1 Geometric parameters of the cascades.

\begin{tabular}{ll}
\hline Parameters & Data \\
\hline Blade chord ( C ) & $146 \mathrm{~mm}$ \\
Aspect ratio (AR) & 1 \\
Blade number & 3 \\
Stagger Angle $(\gamma)$ & $35^{\circ}$ \\
Blade span & $146 \mathrm{~mm}$ \\
\hline
\end{tabular}

\section{Experimental Procedure}

\subsection{Reynolds Number}

At the beginning of each experiment, it was essential to attain reasonably uniform conditions at entry to the cascade. This was obtained by means of adjusting both top and bottom suction rates and, also, the included angle of the bottom diffuser. Given low free-stream turbulence intensity, sufficiently low values of Reynolds number give rise to laminar separation over most of the blade suction surface. This separation may occur at low incidence resulting in high loss [8]. Horlock, Shaw, Pallard and Lewkowicz have shown that there is a minimum value of Reynolds number to have no effect on the performance a conclusion which was reached also by Rhoden [9]. This "critical value" is about 100,000 for the cascade under investigation, The Reynolds numbers of most NGTE low-speed cascade tests, based on blade chords and inlet velocities fall roughly in the range from 150,000 to 500,000 . Because of widely varying turbulence levels of various cascade tunnels, effective Reynolds number is frequently used when data from different-tunnels are being compared. Reynolds number value in the present work was varied from 340,000 to 460,000 based on blade chord length.

The principal effect of wall stall is the destruction of the two-dimensionality of the mainstream and the consequent increase in axial velocity. It is clear that the increase in axial velocity is the predominant cause of the deterioration of the static pressure rise coefficient. The effect of total pressure losses is small until close to stall, when it becomes dominant.

\subsection{Profile Pressure Distribution}

30 pressure tappings were mounted evenly all around the surface of the middle profile both on the pressure and suction surfaces. A suitable data accusation system was used to $\log$ the surface static pressures under different conditions, as shown in Fig. 2.

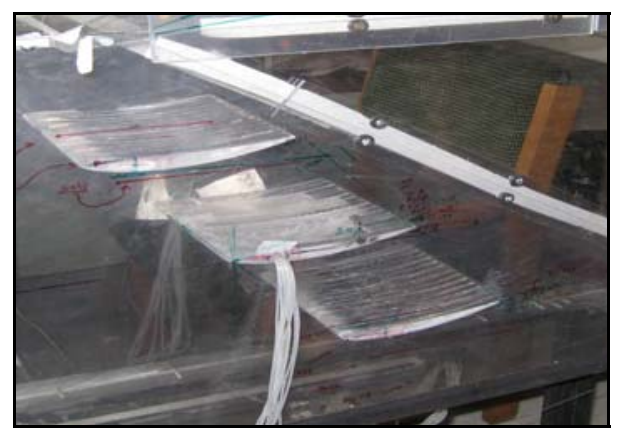

Fig. 1 Cascade set up.

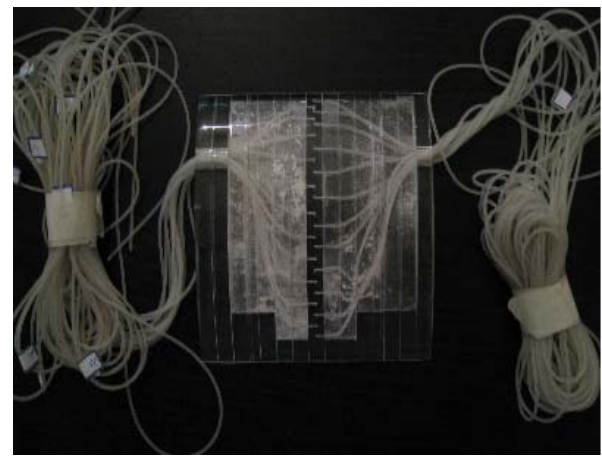

Fig. 2 Pressure tappings configuration.

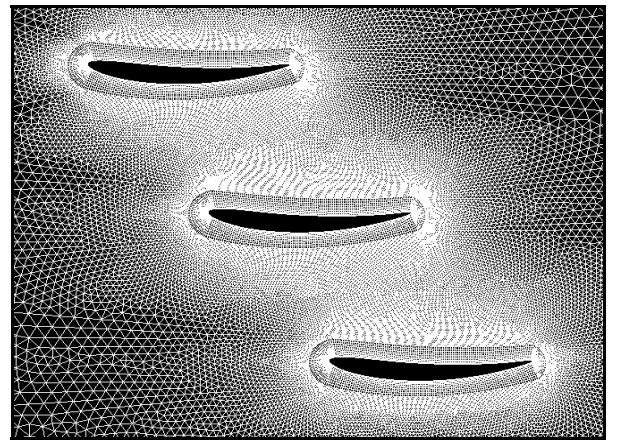

Fig. 3 Computational field.

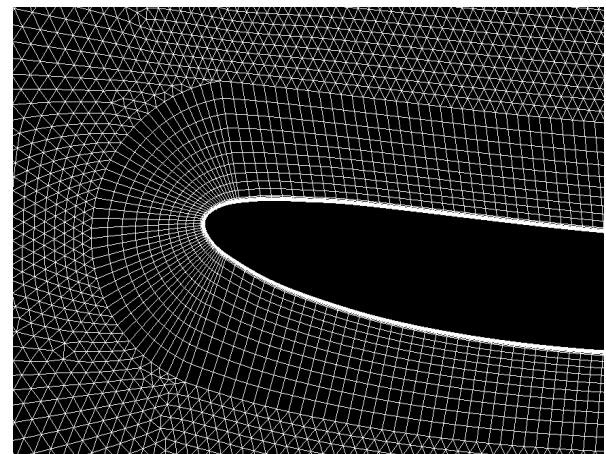

Fig. 4 Geometries and computational mesh structure. 

Surface Pressure Distributions of Axial Compressor Blades

\section{Numerical Solution Method}

Most turbomachinery flows encountered in practice are turbulent and three-dimensional in nature. In addition, turbulence is dissipative and diffusive. As a result, because of the complex nature of the turbulence, especially in compressors, simple turbulence models which are based on simple shear layers fail to capture all turbulence effects. Turbulence can not be modelled by any of the approaches, which utilize the zero, one and simple two-equation turbulence models. The realizable $\mathrm{k}-\varepsilon$ model has different equations from $\mathrm{k}-\varepsilon$ model for simulation of eddy viscosity and turbulent energy dissipation. This kind of turbulence modeling is relatively simple to implement; moreover, it provides superior performance for flows involving boundary layer flows with large adverse pressure gradients, separation and circulation. Since this model accounts for the effects of streamline curvature, swirl, rotation and rapid changes in strain rate in a more rigorous manner than one-equation and other two-equation models, it has greater potential to give accurate predictions for complex flows. The realizable $\mathrm{k}-\varepsilon$ model was designed specifically for turbomachinery applications involving wall-bounded flows and has been shown to give good results for boundary layers subjected to adverse pressure gradients [10].
As normally used for cascade flows, the H-O-H grid system near the blade surfaces is adopted and unstructured grids are used for the flow field which is far away blades. As shown in Figs. 2 and 3, using these grids system allows a fine mesh round the blade (i.e., in the blade passage) and fits the blade shape very well. Near the blade surface, the mesh is set even finer. All these mesh characteristics lead to improved resolution of flow information from the computation, thus are advantageous to better predict boundary layer development.

In realizable $\mathrm{k}-\varepsilon$ model there are new transport Eq.

(8) for turbulent kinetic energy and $\varepsilon$ modeling:

$$
\rho \frac{D K}{D t}=\frac{\partial}{\partial x_{j}}\left[\left(\mu+\frac{\mu_{t}}{\sigma_{k}}\right) \frac{\partial K}{\partial x_{j}}\right]+G_{k}-\rho \varepsilon
$$

$G_{k}$ is turbulent kinetic energy generatin term.

$$
\rho \frac{D \varepsilon}{D t}=\frac{\partial}{\partial x_{j}}\left[\left(\mu+\frac{\mu_{t}}{\delta_{\varepsilon}}\right) \frac{\partial \varepsilon}{\partial x_{j}}\right]+\rho C_{1} S \varepsilon-\rho C_{2} \frac{\varepsilon^{2}}{K+\sqrt{v \varepsilon}}
$$

In this Eq. (9) $C_{1}$ and $C_{2}$ are constant.

\section{Conclusions}

In this paper, the unsteady flow field within an axial cascade of three rotor blades has been investigated with emphasis on the influence of incidence effects on pressure distributions. The results of pressure distribution in terms of pressure coefficient are plotted along the blade chord in difference incidences at various

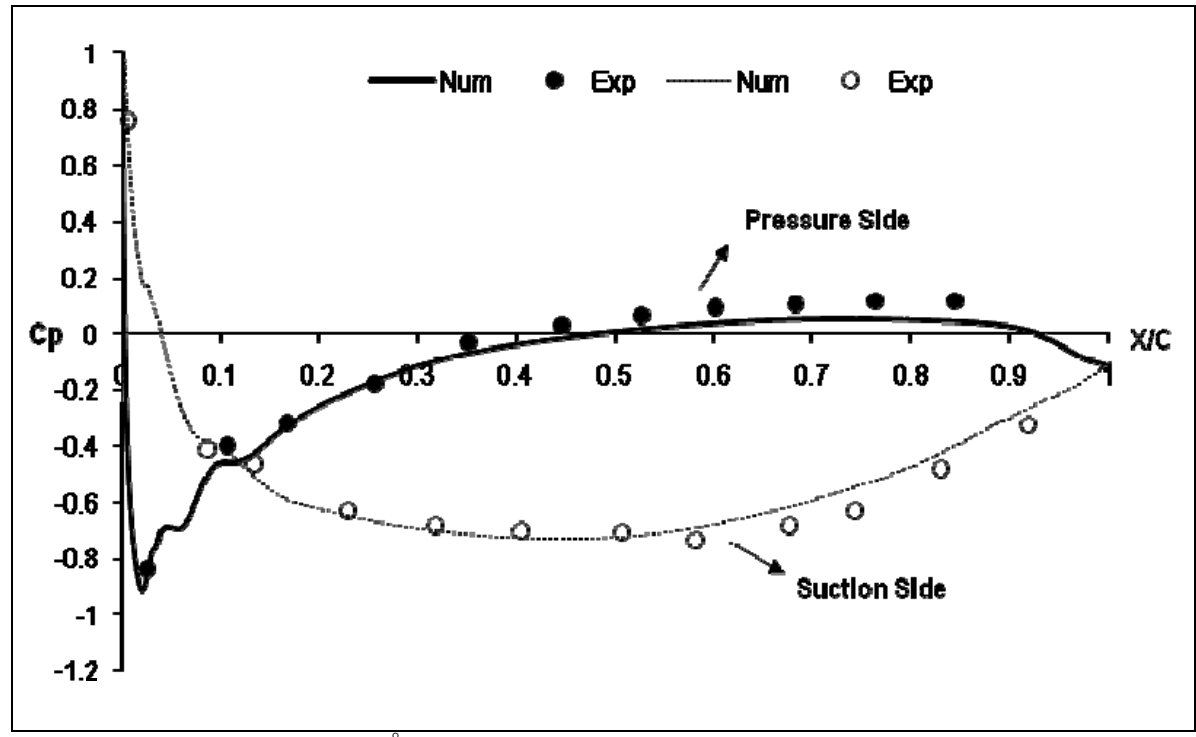

Fig. 5 Pressure distribution in incidence $=\left(-8^{\circ}\right), R e=340,000$ 

Surface Pressure Distributions of Axial Compressor Blades

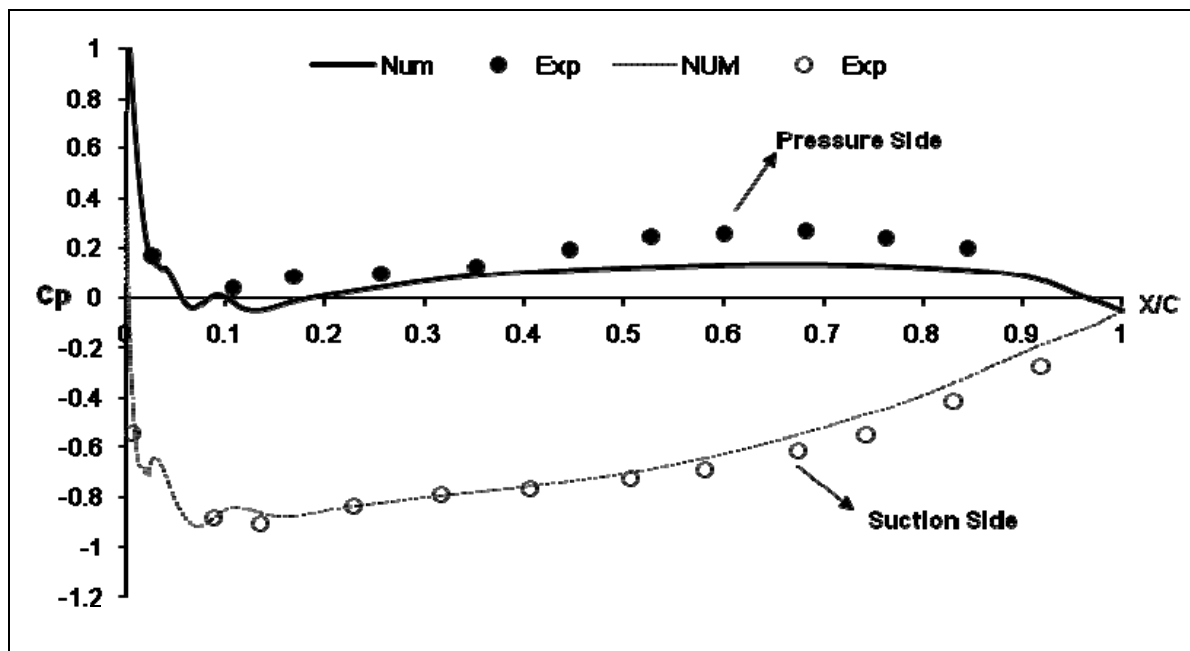

Fig. 6 Pressure distribution in incidence $=\left(2^{\circ}\right), \operatorname{Re}=340,000$

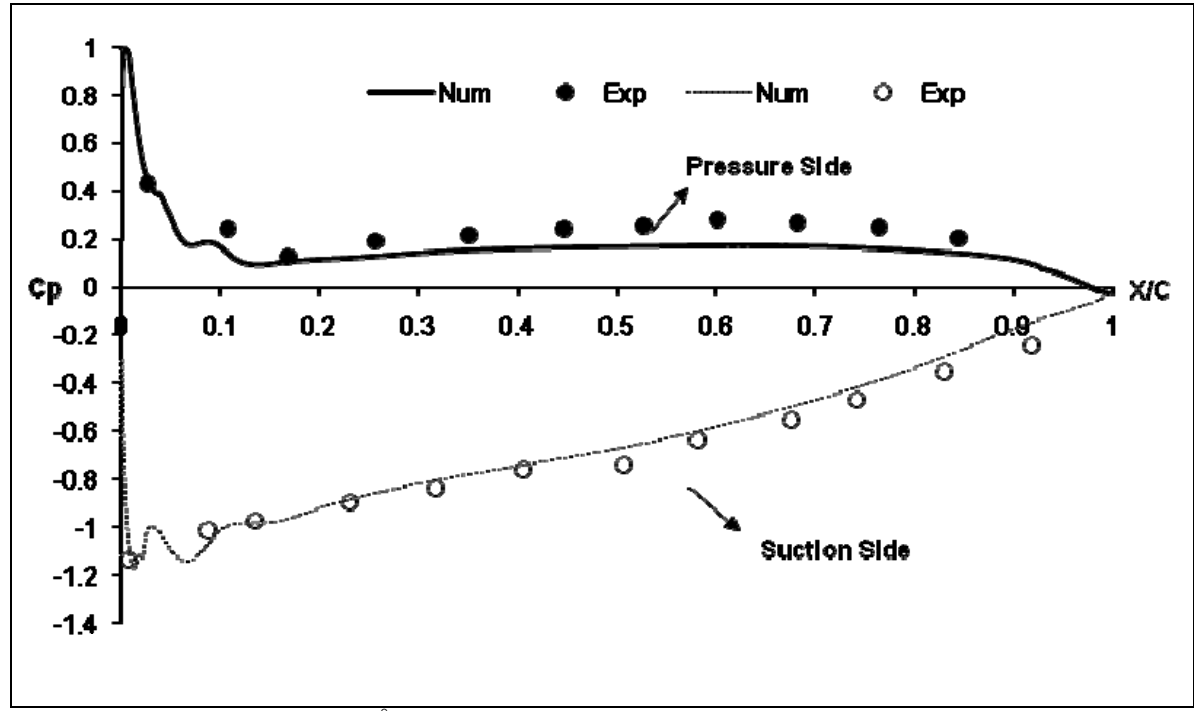

Fig. 7 Pressure distribution in incidence $=\left(6^{\circ}\right), \operatorname{Re}=340,000$.

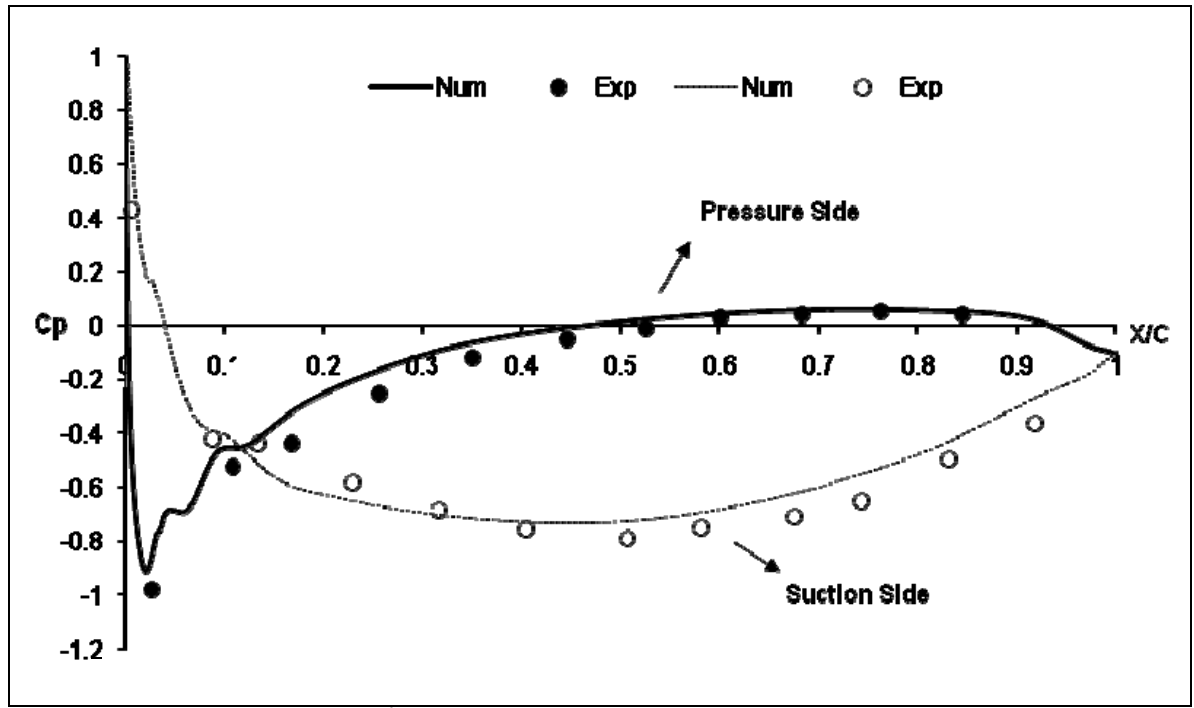

Fig. 8 Pressure distribution in incidence $=\left(-8^{\circ}\right), \operatorname{Re}=460,000$ 

Surface Pressure Distributions of Axial Compressor Blades

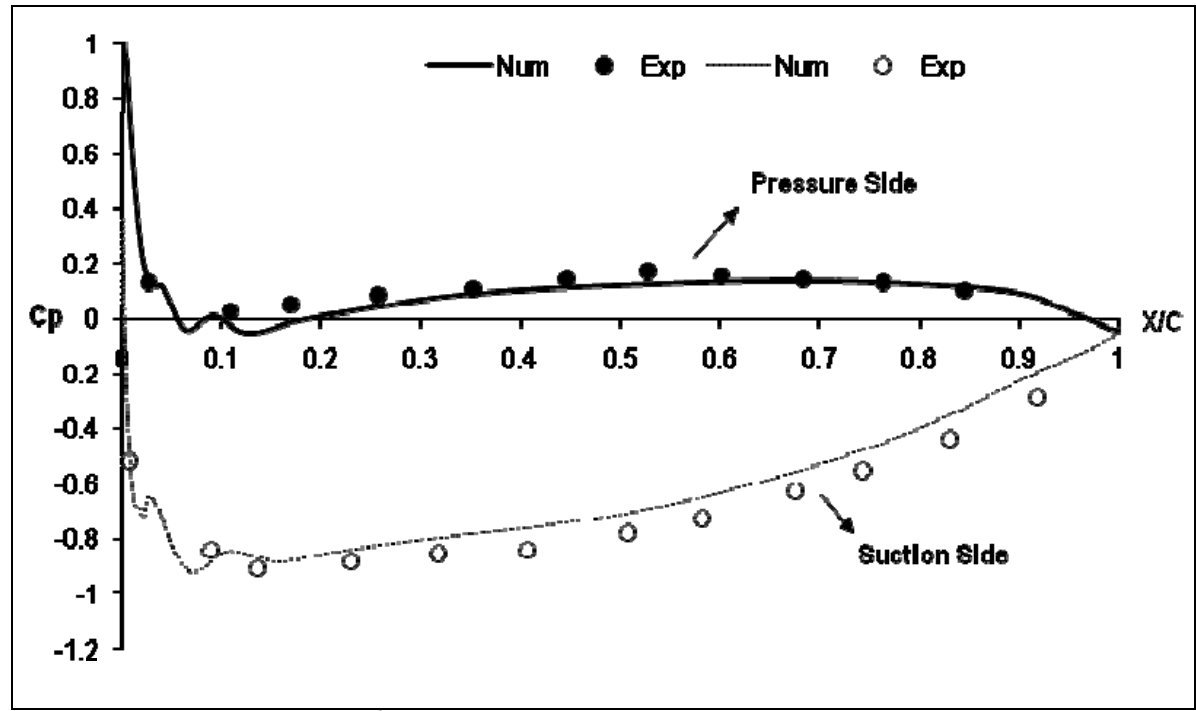

Fig. 9 Pressure distribution in incidence $=\left(2^{\circ}\right) \mathrm{Re}=460,000$.

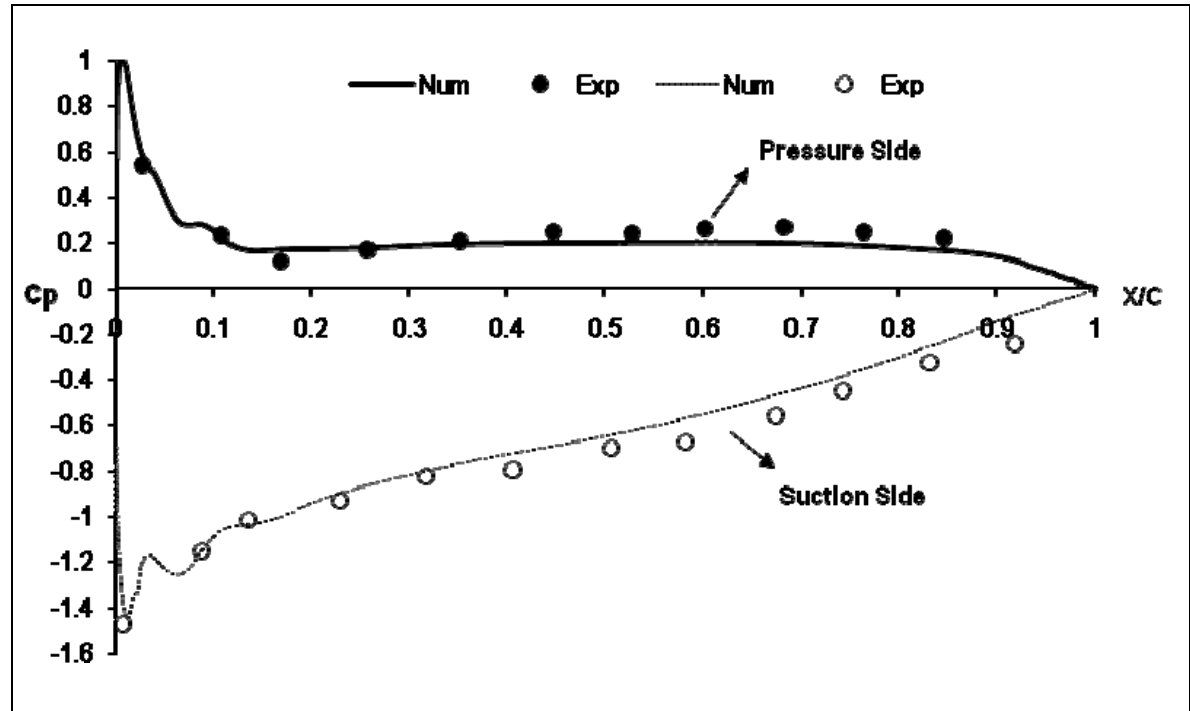

Fig. 10 Pressure distribution in incidence $=\left(8^{\circ}\right), \operatorname{Re}=460,000$.

positions given by ratio $\mathrm{X} / \mathrm{C} \%$. Among the numerous results some limited are selected for presentation and discussion. As it is obvious from the figures on pressure side, the pressure coefficient was getting smaller when the incidence decreases. It was also visible on the suction side that with increasing the incidences $C p$ was getting smaller till $40 \%$ chord and after that this amount was getting larger. It should be mentioned that stagnation point with increasing the incidence move from suction side to pressure side. The pressure coefficient distribution at the two Reynolds numbers can be discussed here. Because of dynamic pressure term in pressure coefficient equation the pressure distribution on both sides of blade in Reynolds number of 460,000 is smaller than 340,000 .

\section{References}

[1] Erwin, R. J., and Yacobi, L. A. 1953. "Method of Estimating the Incompressible Flow Pressure Distribution of Compressor Blade Sections at Design Angle of Attack." NACA, RML 53F 17.

[2] Aerodynamic Design of Axial-Flow Compressors, revised, NASA SP-36, 1965.

[3] Howell, A. R. 1947. "Design of Axial Compressors, Lectures on the Development of the British Gas Turbine Jet Unit." War Emergency 452-462.

[4] Kus, U., and Chauvin, J. 1995. "Performance Prediction of Turbomachinery Cascade as Affected by Upstream 
Turbulence.” Journal of physical III 5 (10): 1599-1620. (in French)

[5] Yahya, S. M. 2003. "Turbine Compressor and Fans." 2ed Ed., Tata McGraw-Hill Education.

[6] Rae, W. H., and Pope, A. 1984. Low-Speed Wind Tunnel Testing. SE: John Wiley \& Sons.

[7] Turton, R. K. 1995. Principles of Turbomachinery. Second Edition, Chapman \& Hall.

[8] Lieblein, S. 1965. "Experimental Flow in
Two-Dimensional Cascades." In Report SP-36 6: 183-226.

[9] Horlock. 1985. "Experimental Investigation of Losses and Secondary Flow in an Axial Compressor Stage." Engineering Research 51 (2): 33-40. (in German)

[10] Lakshminarayana, B. 1992. "An Assessment of Computational Fluid Dynamic Techniques in the Analysis and Design of Turbomachinery." ASME Journal of Fluids Engineering 113: 315-352. 